\section{THE AUTHOR FILE}

\section{Markus Sauer}

\author{
Skiing his way to multiplexed imaging.
}

Music offers a kaleidoscope of impressions; paintings are often a symphony of color. Microscopy images are

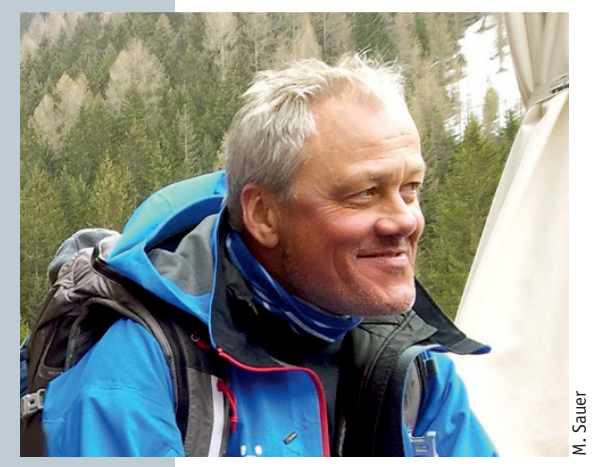

Markus Sauer this rich, too, but the aesthetic pleasure can give way to a certain scientific wistfulness. To study multiple interactions in the cell, labs would like to spectrally resolve more than the four or five fluorophores that filters currently help them to identify.

Markus Sauer, a physical chemist and super-resolution microscopist at the University of Würzburg, and his team, have now labeled 12 different cellular targets with highly similar dyes and identified them with three lasers and 32 spectral channels. The approach distinguishes the fluorophores according to their emission wavelength and fluorescence lifetime. Each hue has an unequivocally identifiable signature, says Sauer.

The team chose and tested green, red and yellow dyes for the spectroscopic behavior, using three lasers to excite each probe. Sauer was happy to see how constant the spectroscopic signatures and lifetimes remained in the somewhat unpredictable cellular environment. It was a surprise that each dye's behavior is influenced only by its immediate neighborhood-such as the amino acids of the antibody to which it is linked. But, he says, "the dye does not notice the other cellular molecules." This makes the dye's behavior reliable wherever it is in the cell.

The lab developed an algorithm that can detect individual signals just as a trained ear can hear individual instruments playing a symphony. The pattern-matching algorithm is discerning: it can identify a difference in emission wavelength of one to two nanometers or a variation in fluorescence lifetime of a few tens of picoseconds. The algorithm was trained on each individual signal, which allows individual fluorophores to be picked out of the color blend.

There has not been any one moment at which to shout 'hurrah'; Sauer and his group spent the past two years incrementally improving the labeling and the algorithm to achieve results that were, as he says, pretty enough.

This new method for spectrally resolved fluorescence lifetime imaging microscopy and its application to stimulated emission depletion microscopy stems

from Sauer's long-standing interest in exploring how to influence dye photophysics. He has spent much of his career in super-resolution microscopy. His group developed direct stochastic optical reconstruction microscopy, or dSTORM, a method to localize single molecules that lets labs do super-resolution imaging with standard fluorescent probes. "dSTORM is close to my heart," says Sauer. Over three-quarters of his group work on dSTORM.

Sauer received his $\mathrm{PhD}$ in physical chemistry at the University of Heidelberg and then did a fellowship at Lawrence Berkeley National Lab to learn more about quantum dots, nanocrystals and single-molecule energy transfer. He obtained the additional Dr. habil degree in Heidelberg and was offered a professorship at the University of Bielefeld. He taught experimental physics, which, he jokes, meant he had to "study" physics. In 2009 he joined the faculty of the University of Würzburg, where he teaches biophysics to physicists, chemists and biologists. An award, the BioFuture Prize, allowed him to start his own group, and he recently received an award at the Biophysical Society's annual meeting.

Sauer also cofounded ATTO-TEC, a company that sells fluorescent dyes and does custom research. He is not actively involved in the company but is happy that, after many challenging years, it is doing well. He has 15 people in his lab and runs four addi-

"Skiing is an addiction." tional lab groups in the department. Keeping hierarchical structures low is his management approach, he says. Instead of pressure, he practices active encouragement. The group gets together outside the lab for barbeques and to play soccer, and there is an annual ski seminar in the Alps, now in its 14th year. "We have talks every evening but the day is reserved for skiing," says Sauer. He escapes to the slopes when he can-at least four times a winter. "Skiing is an addiction," he says. He also plays the guitar and trumpet but has found too little time to do so lately.

Sauer's viewpoint as a chemist is unique in the superresolution microscopy field, says Luke Lavis, a researcher at the Howard Hughes Medical Institute's Janelia Research Campus who collaborates with Sauer. Sauer's deep thoughts about the behavior of molecules led to dSTORM with single fluorophore labels. His work on electron transfer in fluorophores was so rigorous and careful that Lavis expected Sauer to be boring. "Boy was I wrong," says Lavis. "Markus is, in a word, cool," he says. "But behind his fun and dynamic personality is a razor-sharp mind that integrates the electronic behavior of molecules and the ultrastructure of cells."

\section{Vivien Marx}

Niehörster, T. et al. Multi-target spectrally resolved fluorescence lifetime imaging microscopy. Nat. Methods 13 257-262 (2016). 